\title{
Numerical Simulation of the Effect of Respiratory Cycle on Inhalable Particle Deposition in Alveolus
}

\author{
Jun Jin, Jianren Fan \\ College of Energy Engineering, Zhejiang University \\ 38, Zheda Road, Hangzhou, China \\ jinj@zju.edu.cn; fanjr@zju.edu.cn
}

\begin{abstract}
After being inhaled into the human respiratory tract, some of the inhalable particles in the air do not deposit in the respiratory tract conduction trachea, instead, they deposit deeper into the respiratory alveolar region, which results in many diseases such as bronchitis, emphysema and asthma. Meanwhile, nebulizer medicine is increasingly being adopted to the treatment against respiratory diseases. Therefore, it is very important to understand the characteristics of the airflow structure, the movement, dispersion and deposition of aerosols in the gas exchange region. In this paper, we adopt the computational fluid dynamics (CFD) method to study the effect of the respiratory cycle on the transport and deposition of inhalable particles in the alveolar system. We use the direct numerical simulation (DNS) method to calculate the continuous phase, while the particle phase is traced by Lagrange method.
\end{abstract}

Keywords: Respiratory Cycle, Inhalable Particle, Alveolus, Numerical Simulation.

\section{Introduction}

Inhalable particles have close relationship with the health of respiratory system [1-2]. We investigate the effect of respiratory cycle on inhalable particle deposition in alveolus numerically in this paper. The particle deposition behavior and characteristics of three cases of different respiratory cycles have been simulated. $\mathrm{T}=1.0 \mathrm{~s}$ represents the respiratory cycle during strenuous exercise, $\mathrm{T}=2.4 \mathrm{~s}$ represents the daily life respiratory cycle, and $\mathrm{T}=4.0 \mathrm{~s}$ represents the respiratory cycle during sleep. The corresponding breathing volume is $60 \mathrm{~L} / \mathrm{min}, 30 \mathrm{~L} / \mathrm{min}$, and $15 \mathrm{~L} / \mathrm{min}$ respectively. The period and phase of the mainstream variation in the model are as the same as that of the contraction and expansion of the alveolus. The smaller the respiratory cycle, the larger the frequency of the mainstream in the alveolar duct, also the faster the contraction and expansion of the alveoli.

\section{Results and Discussion}

\subsection{The Effect of the Respiration Cycle on the Transmission Characteristics of Particles}

Fig. 1 is the transient distribution of the particles of $d P=1.0 \mu \mathrm{m}$ under three respiratory cycles.

We can see that when $t=25 / 24 \mathrm{~T}$, the "W" profile of the transient particle distribution in the alveolar duct is becoming flat gradually with the increase of the respiratory cycle. This indicates that the smaller the respiratory cycle, the larger the fluid velocity near the wall of the alveolar tube at this transient time. Because if the respiratory cycle is small, the alveolar dilatation becomes large during the limited time, therefore the axial velocity of the fluid near the wall of the alveolar tube becomes large, too.

When $\mathrm{t}=5 / 4 \mathrm{~T}$, although all of the particle distributions in the tube are parabolic for the three respiratory cycles, the larger the respiratory cycle, the more steep the top of the parabola. This is because the larger the respiratory cycle, the slower the mainstream velocity attenuates. At this transient time, the mainstream velocity in the center of the alveolar tube is the largest for the $\mathrm{T}=4.0 \mathrm{~s}$ condition.

When $\mathrm{t}=73 / 48 \mathrm{~T}$, the transient particle distributions are as identical as each other for the three respiratory cycles, all of them appear to be disordered.

When $\mathrm{t}=7 / 4 \mathrm{~T}$, most of the particles have disappeared from the computational domain, there are only a few particles left in the alveolar cavity along the gravity direction. Moreover, the larger the respiratory cycle, the more residual particles in the alveolus. 


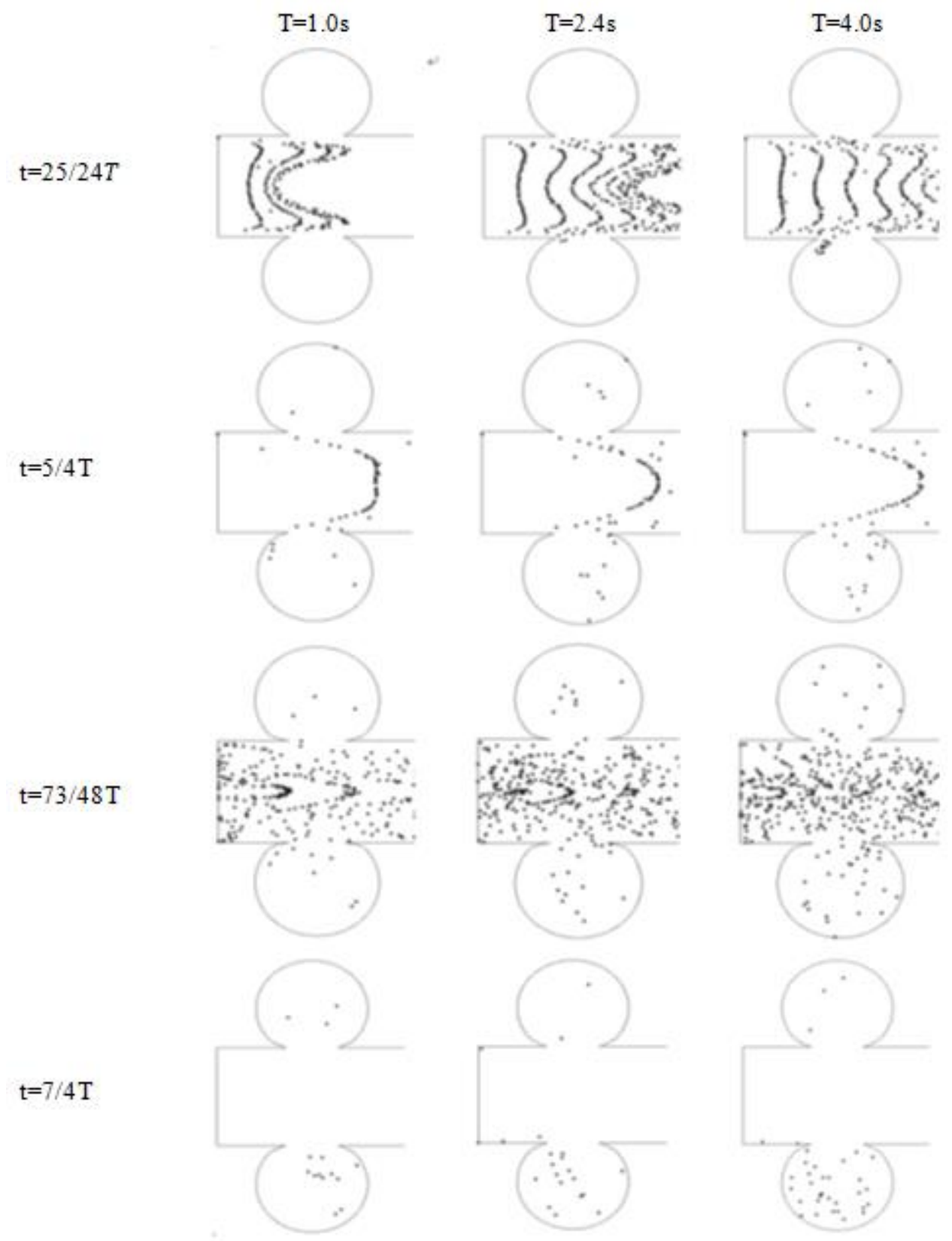

Fig. 1: The transient distributions of the particles of $d P=1.0 \mu m$ under three respiratory cycles.

We find the respiration cycle has a significant influence on the transient distribution of the medium-size particles $(1.0 \mu \mathrm{m} \sim 2.0 \mu \mathrm{m})$. Because of the larger the respiratory cycle, the more slowly the mainstream velocity decays, which most significantly influences the medium size particles that are mainly affected by the flow drag effect. 


\subsection{The Effect of the Respiration Cycle on the Particle Deposition}

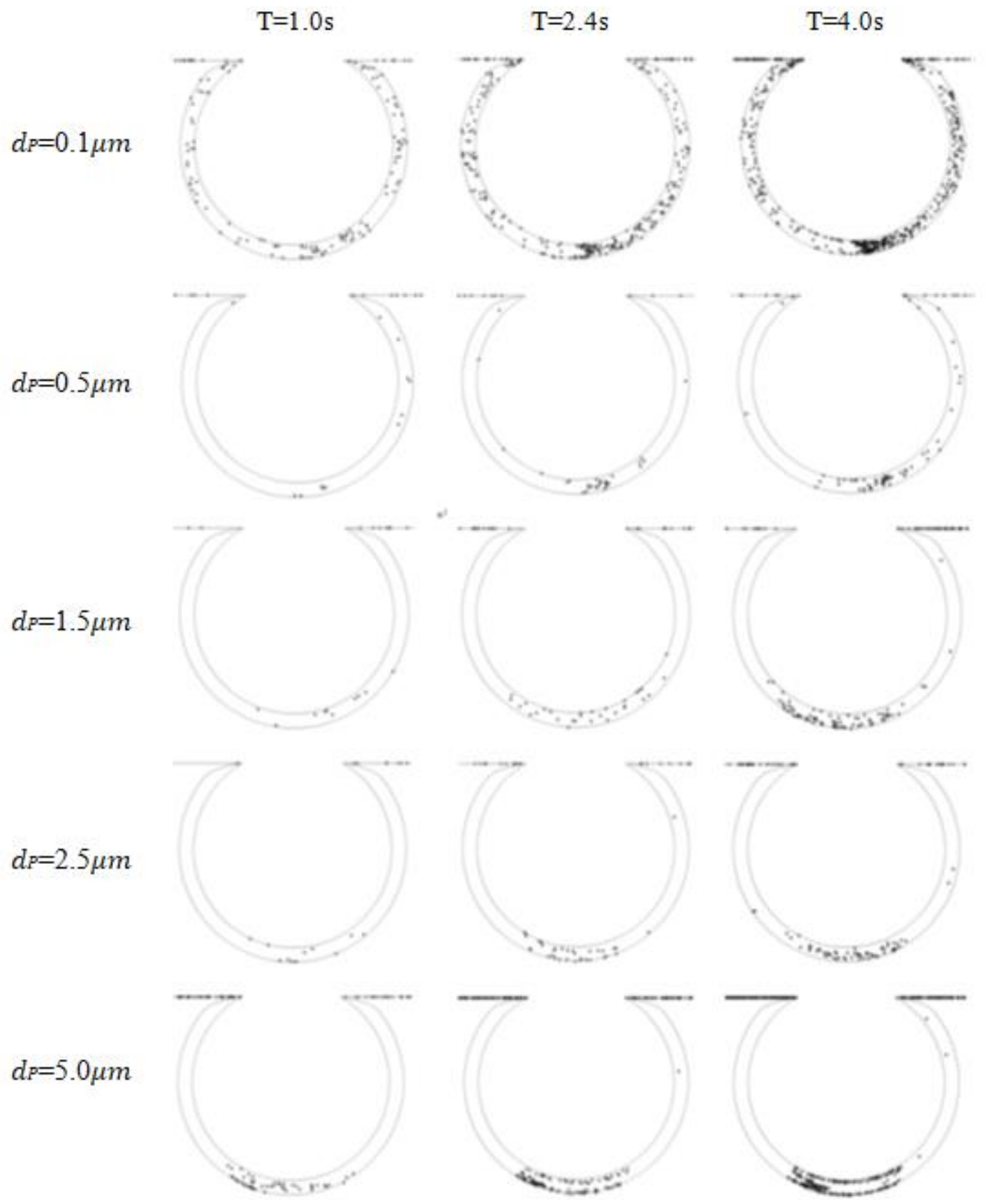

Fig. 2: The particle deposition distributions under three respiratory cycles.

Fig. 2 is the result of the deposition distributions of multi-size particles for three respiratory cycles. We can find that the deposition distribution characteristics of the particles of various size mentioned in this paper are almost the same among three cases of different respiratory cycles, which indicates that the respiratory cycle does not have a significant effect on the deposition distribution of the particles. The only difference is that the greater the respiratory cycle, the more particles that are injected into the computational domain at the inhalation stage, thus the more particles deposited in the alveoli. 


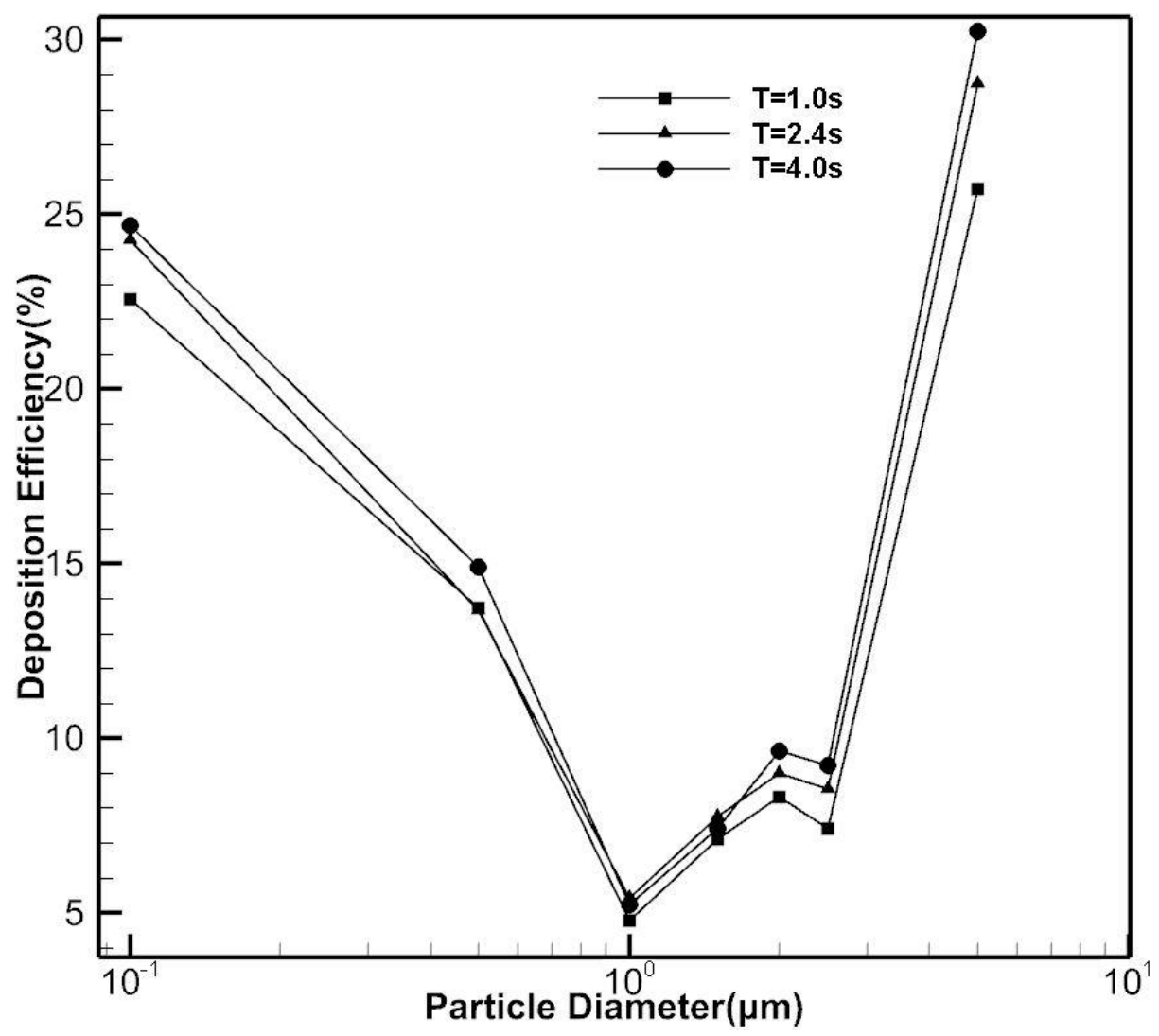

Fig. 3: The deposition efficiency of particles under three respiratory cycles.

Fig. 3 is a line chart of the deposition efficiency of particles under three respiratory cycles.

As we see from Fig. 3, with the increase of particle size, the deposition efficiency of the particles decreases at first and increases thereafter. The deposition efficiency of particles with a diameter of $1.0 \mu \mathrm{m}$ is the smallest, which is about $5 \%$. While that of the particles with a diameter of $5.0 \mu \mathrm{m}$ is found to be the largest. The deposition efficiency is about $31 \%, 27 \%$ and $26 \%$, for the respiratory cycle $\mathrm{T}=4.0 \mathrm{~s}, \mathrm{~T}=2.4 \mathrm{~s}$ and $\mathrm{T}=1.0 \mathrm{~s}$ respectively.

On the one hand, in case of $\mathrm{T}=4.0 \mathrm{~s}$, the deposition efficiency in the alveolar of the particles of all sizes are more or less higher than that for the other two respiratory cases. In case of $\mathrm{T}=2.4 \mathrm{~s}$, the deposition efficiency in the alveolar of the particles of all sizes are more or less higher than that for the $\mathrm{T}=1.0 \mathrm{~s}$ case. This obviously indicates that the larger the respiratory cycle, the higher the particle deposition efficiency. But it should also be noticed that the maximum difference is $5 \%$ only.

On the other hand, we can assume that the particles are equally distributed in the air and the particle density is $\alpha$. Because the corresponding breathing volume for $\mathrm{T}=1.0 \mathrm{~s}, \mathrm{~T}=2.4 \mathrm{~s}$ and $\mathrm{T}=4.0 \mathrm{~s}$ are $60 \mathrm{~L} / \mathrm{min}, 30 \mathrm{~L} / \mathrm{min}$, and $15 \mathrm{~L} / \mathrm{min}$ respectively, the total number of particles entering the computational domain within one minute for $\mathrm{T}=1.0 \mathrm{~s}, \mathrm{~T}=2.4 \mathrm{~s}$ and $\mathrm{T}=4.0 \mathrm{~s}$ are $60 \alpha, 30 \alpha$ and $15 \alpha$, respectively.

To sum up, the result is that the entire particles deposited in the alveolar domain for $\mathrm{T}=1.0 \mathrm{~s}$ case is far larger than the other two cases. Moreover, the larger the number of particles deposited in the alveolar domain, the more harmful the damage to the alveolar system. Therefore, the smaller the respiratory cycle, the more inhalable particles deposited in the alveoli, which will cause more serious damage to the respiratory system.

\section{Conclusion}

The respiration cycle has a significant influence on the transient distribution of the medium-size particles. The respiratory cycle does not have an obvious effect on the deposition distribution of the particles. 
With the increase of the particle size, the particle deposition efficiency decreases at first and increases thereafter. Taking into account the breathing volume, the smaller the respiratory cycle, the more entire particles deposited in the alveoli.

\section{Acknowledgements}

The authors are grateful to the support of the National Natural Science Foundation of China (No. 51376161) to this work.

\section{References}

[1] B. Grgic, W. H. Finlay, A. F. Heenan, "Regional aerosol deposition and flow measurements in an idealized mouth and throat," J. Aerosol Sci., vol. 35, pp. 21-32, 2004.

[2] Y. S. Cheng, Y. Zhou, B. T. Chen, "Particle deposition in a case of human oral airway," Aerosol Sci. and Tech., vol. 31, pp. 286-300, 1999. 